\title{
SONG - Stellar Observations Network Group
}

\author{
Frank Grundahl $^{1}$, J. Christensen-Dalsgaard ${ }^{1}$, H. Kjeldsen ${ }^{1}$, \\ S. Frandsen ${ }^{1}$, T. Arentoft ${ }^{1}$, P. Kjaergaard ${ }^{2}$ and U. G. Jørgensen ${ }^{2}$ \\ ${ }^{1}$ Danish AsteroSeismology Centre, Department of Physics and Astronomy, University of \\ Aarhus, DK-8000 Aarhus C, Denmark \\ email: fgj@phys.au.dk \\ ${ }^{2}$ Niels Bohr Institute, Copenhagen University, Juliane Maries Vej 30, \\ DK-2100 Copenhagen, Denmark
}

\begin{abstract}
Several areas of stellar observations depend critically on nearly continuous observations of individual objects over very extended periods. Important examples are investigations of stellar oscillations to carry out asteroseismology, and the search for extra-solar planets. To meet this requirement we are establishing the SONG network, consisting of 8 sites with a 1-meter-class telescope with a suitable geographical distribution. These will be optimized for asteroseismology based on Doppler-velocity observations and the characterization of extra-solar planets with photometry, using gravitational microlensing. Funding has been obtained towards the construction of the prototype SONG telescope which will be set up on Tenerife, with first light expected in 2011. The full network will be established in parallel with the tests of the prototype and is planned to be operational in 2014.
\end{abstract}

Keywords. instrumentation: spectrographs, techniques high angular resolution, stars: oscillations, stars: planetary systems

\section{Asteroseismology}

During the past $\sim 25$ years helioseismology has proven itself as an extremely valuable tool for studying the detailed properties of the solar interior; it is, however, only during the past $\sim 5$ years that asteroseismology - seismology of stars other than the Sun - has become possible. This is due to the large progress in measuring precise stellar radial velocities achieved by the groups searching for extra-solar planets via the radial velocity method. Solar-like oscillations in stars manifest themselves as low-amplitude radial velocity variations due to the surface motions imposed by the oscillations.

In order to measure stellar oscillation frequencies to high precision and to separate closely spaced pairs of frequencies it is necessary to obtain long and continuous radialvelocity time series. At present it is very difficult to obtain time strings longer than around two weeks. This is the motivation for building a network of telescopes.

For asteroseismology SONG will be able to observe solar-like oscillations in stars brighter than $V=6$ and thus obtain their oscillation spectra. Measuring the oscillations using radial velocities has a very substantial advantage over intensity observations since the background signal (noise) from the star is much lower (Grundahl et al. 2007).

To measure the oscillations the stars will be observed with a high-resolution spectrograph equipped with an iodine cell for velocity reference (Butler et al. 1996). For bright stars this is expected to reach precisions of $1 \mathrm{~m} / \mathrm{s}$ per minute of observation. A prediction of the expected velocity precision is shown in figure 1 . 




Figure 1. The radial velocity presision as a function of stellar magnitude for a $1 \mathrm{~m}$ SONG telescope. The calculation is for a slowly rotating star resembling $\alpha$ Cen A.

\section{Microlensing planet search}

The main science driver for our exoplanet search is to be able to characterize the population of habitable exoplanets in our Galaxy. A planet is habitable if it is large enough to maintain an atmosphere, small enough to not transform itself into a gas planet, and in the right orbital distance from its star to house liquid water at its surface.

SONG will detect small planets using both the microlensing technique and Doppler velocity method. Recently the microlensing method has demonstrated the capability to detect low-mass planets (Beaulieau, et al. 2006; Bennett, et al. 2008), and for SONG it is expected that it will be possible to detect low-mass planets over a wider range of orbital periods than for the Doppler method (for further details, see Jørgensen 2008). The combination of long time series and high-resolution photometry and spectroscopy allows SONG to identify exoplanets in orbits all the way from far inside Mercury's orbit, to Neptune-like orbits and beyond. SONG will be able to detect habitable exoplanets around all types of stars, and for many orbital radii SONG may be able to detect planets as small as Mars (Jørgensen 2008).

The design of SONG is made such that within 5 years of observation we will be able to either characterize the Earth-like exoplanet population, or (in case of non-detection) will be able to put a $5 \%$ upper limit on the existence of Earth mass exoplanets around the weighted-average population of stars in our Galaxy.

\section{References}

Beaulieu et al. 2006, Nature 439, 437

Bennett et al. 2008, ApJ, in the press (arXiv:0806.0225)

Butler, R. P., Marcy, G. W., Williams, E., McCarthy, C., Dosanjh, P., \& Vogt, S. S. 1996, PASP, 108, 500

Grundahl, F., Kjeldsen, H., Christensen-Dalsgaard, J., Arentoft, T., \& Frandsen, S. 2007, CoAst 150,300

Jørgensen, U. G. 2008, Phys. Scr., T129, in the press 